
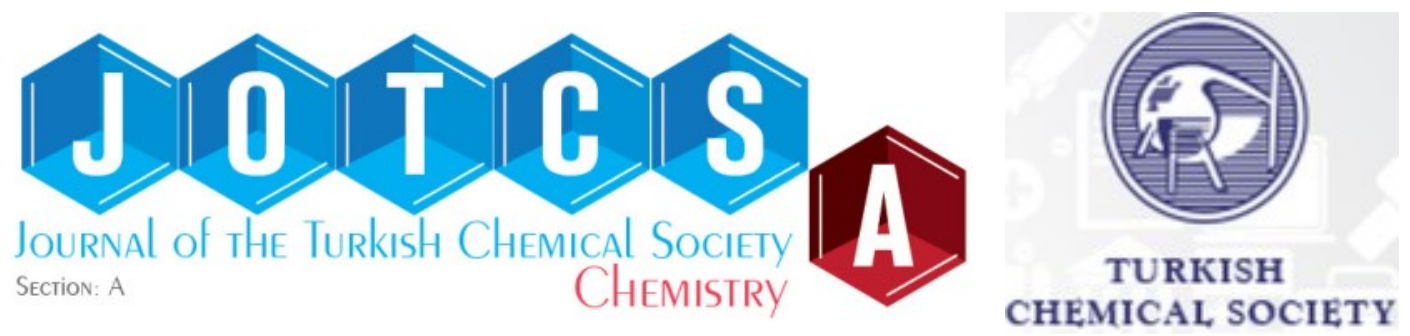

\title{
Effect of Temperature on Stability of Lipid Microbubbles
}

\author{
Sevgi Kilic* \\ Department of Chemical Engineering, Izmir Institute of Technology, Urla, 35430-Izmir, TURKEY
}

Abstract: The effect of temperature on stability of lipid microbubble shell containing polyethyleneoxide-40stearate (PEG ${ }_{40} \mathrm{St}$ ) as emulsifier was investigated. Microbubbles at $4{ }^{\circ} \mathrm{C}$ were subjected to different temperatures up to $48{ }^{\circ} \mathrm{C}$ (down-to-up) and it was found that both the number and the size of microbubbles remained unchanged in the population up to a certain time, so called "onset time". The onset time was about 6 hours at $10^{\circ} \mathrm{C}, 2$ hours at $20^{\circ} \mathrm{C}$ and shorter at elevated temperatures, exhibiting an exponential decrease with increasing temperature. Once the onset time was reached, the number of microbubbles started to decrease and the average size of the population started to increase. Observation of single microbubbles on a constant temperature heating stage exhibited that each microbubble had its own onset time, with the smaller microbubbles vanishing earlier than the larger ones. The Langmuir monolayer studies showed that hydration degree of the emulsifier PEG chains decreased with temperature, causing them go through conformational changes and subsequently destabilization of the shell. By subjecting the freshly produced microbubbles directly to the desired temperatures in up-to-down fashion, more stable microbubbles were able to be produced, with their onset time increased $40 \%$ at $10^{\circ} \mathrm{C}$ to $500 \%$ at $38^{\circ} \mathrm{C}$. Overall, the results suggest that the new strategies need to be developed to control the collapse process in the microbubble shell resulting from the conformational changes in the PEG chains of the emulsifier for the design of more stable microbubbles.

Keywords: Microbubble, ultrasound contrast agent, Langmuir monolayers, temperature, phospholipid, emulsifier, PEG conformations, stability

Submitted: July 19, 2019. Accepted: October 01, 2019.

Cite this: Kilic S. Effect of Temperature on Stability of Lipid Microbubbles. JOTCSA. 2019;6(3):439-54.

DOI: https://doi.org/10.18596/jotcsa.594219.

*Corresponding author. E-mail: sevgikilic@iyte.edu.tr. Phone: +90-232-750 6647; Fax:+90-232-750 6645.

\section{INTRODUCTION}

Microbubbles, micron-size gas bubbles surrounded by a lipid-based monolayer shell, are contrast agents used in ultrasound to improve the image quality. Microbubbles are also used in targeted gene/drug delivery, metabolic gas transport, and hyperthermia therapy (1). Attachment of therapeutic agents to microbubbles has become one of the popular strategy to achieve more effective targeting, combined with diagnosis and therapy (2). The clinical application of microbubbles with a wide range of applications is however hampered by their restricted lifetime after their administration. Therefore, development of persistent and stable microbubbles has become one of the most promising fields in medicine in order to enhance the therapeutic and diagnostic capabilities of ultrasound. Microbubble persistency is generally related to the dissolution of the gas core, which is associated with the shell permeation resistance (3-8). It is believed that the Laplace driven dissolution of gas core of lipid microbubbles causes changes in the shell structure through buckling and folding process, followed by the collapse of the shell leading to further dissolution of microbubbles (6, 9-14). However, although temperature can have important effect on the molecular orientation and arrangement of the components in a monolayer $(15,16)$, its effect on the shell microstructure has not been investigated truly. The studies mostly focused on temperature dependent behavior of microbubbles. For instance, 
Mulvana et. al showed that commercial SonoVue ${ }^{\circledR}$ microbubbles exhibit a size dependent dissolution rate, with smaller size microbubbles dissolving at higher rates and increasing with increasing temperature at static and acoustically driven conditions (17). They also showed that SonoVue ${ }^{\circledR}$ microbubbles exhibit an increase in the mean bubble diameter of the population with increasing temperature and a decrease in bubble concentration. Through their investigations on the dynamic behavior of individual SonoVue ${ }^{\circledR}$ microbubbles, they demonstrated that the majority of the microbubbles oscillated approximately spherically at room temperature, while the same size bubbles oscillated more violently at body temperature (18). Swanson et al. monitored the change in average size of the population of oxygen microbubbles of both DSPC and DPPC with $\mathrm{PEG}_{40} \mathrm{St}$ and observed that both microbubble type have a tendency to grow in size when stored at $4{ }^{\circ} \mathrm{C}$, with the DPPC coated microbubbles exhibiting smaller change in the mean diameter (19). For the microbubbles composed of DPPC and PEG $_{40}$ St, Farook et al reported that while the initial sizes of the microbubbles remained unchanged for almost 2.5 hours at room temperature, the mean size of the microbubbles decreased rapidly from $6.6 \mu \mathrm{m}$ to $1.3 \mu \mathrm{m}$ in less than an hour at the body temperature (20). Kwan et al monitored the dissolution behavior of a single microbubble composed of various phosphocholine lipids and $\mathrm{PEG}_{40} \mathrm{St}$ as the emulsifier, and filled with $\mathrm{SF}_{6}$ gas, in an air saturated solution and observed that the microbubbles with longer acyl chain length are more persistent at lower temperatures $(5,9)$. They reported that the use of different emulsifiers, for instancemDSPE-PEG 2000 versus PEG $_{40}$ St, did not significantly affect the dissolution behavior of the microbubbles (9). In all these studies, the less stable behavior of the microbubbles with increasing temperature was attributed simply to the changes in the physical properties of the phospholipid shell, which are believed to be determined by the strength of attractive hydrophobic and van der Waals interactions between the hydrocarbon tails of the phospholipid molecules $(9,11,17,21,22)$. In this respect, considerable amount of research was carried out to investigate the effect of lipid hydrocarbon tail length on microbubble stability at different temperatures $(9,13,14,21)$. Unfortunately, the aforementioned studies still remain insufficient to enlighten the mechanism causing destabilization of the shell under temperature and to provide a roadmap for the design of more stable microbubbles. The study investigating the effect of cooling rate on microbubbles microstructure is very challenging, evoking that some other factors as well as hydrophobic and van der Waals interactions may be effective in the shell gas permeability (14). So far, no attention was paid to the role of temperature on emulsifier, which is the crucial component in microbubble formation and possesses bulky polyethylene glycol (PEG) chain. In the present study, along with monitoring the microbubbles dissolution behavior at various temperatures, Langmuir monolayer measurements were performed to reveal the changes in the PEG chain of the emulsifier with temperature. The results showed that the conformational changes induced by temperature in the PEG chain of the emulsifier affect the packing density of microbubble shell and thus has important influence on destabilization of microbubbles.

\section{MATERIALS AND METHODS}

\section{Materials}

1,2-Distearoyl-sn-glycero-3-phosphocholine (DSPC) was purchased from Avanti Polar Lipids Inc (Alabaster, AL). Polyoxyethylene-40 stearate (PEG ${ }_{40} \mathrm{St}$ ), propylene glycol (PG) and chloroform were all purchased from Sigma-Aldrich (St. Louis, MO). All chemicals were used as received.

\section{Preparation of Microbubbles}

A mixture of DSPC and $\mathrm{PEG}_{40}$ St at a molar ratio of 9:1 was dissolved in chloroform. The solution was dried under nitrogen gas and kept in a vacuum oven at room temperature overnight to form completely dried lipid/emulsifier film. The resulting film was then hydrated for 2 hours in phosphate buffer saline (PBS)-propylene glycol (PG) solution mixed at volume ratio of $4: 1$ at $60{ }^{\circ} \mathrm{C}$. The lipid/emulsifier mixture was first sonicated using a probe tip sonicator (Misonix, S4000, Newtown, CT) with the probe tip down in the solution to obtain well dispersed solution. The homogenous mixture was then sonicated continuously for 30 seconds with the tip of the probe at the interface of the air-aqueous solution to form microbubbles. The resulting suspension was diluted with cold PBS at $4{ }^{\circ} \mathrm{C}$. After homogenization, the microbubble solution was aliquoted into $2-\mathrm{mL}$ tubes. Free remaining lipids in the solution were removed via washing of microbubble mixture with cold phosphate buffer solution (PBS) and then centrifugation operated at $2000 \mathrm{rpm}$ for 2 minutes. The infranatant part of the suspension consisting of residual lipids and vesicles were discarded. The resulting cake was resuspended in the cold PBS and centrifuged again. To the final cake in each tube was added PBS:PG $(4: 1)$ mixture up to $1 \mathrm{~mL}$ volume. All tubes were combined in a large syringe to get a homogenous mixture and then the microbubble solution was aliquoted into small vials and stored at $4{ }^{\circ} \mathrm{C}$. All analyses were performed on the next day following storage at $4{ }^{\circ} \mathrm{C}$.

Effect of Temperature on Microbubble Stability Temperature responses of a population of microbubbles were investigated at $4^{\circ} \mathrm{C}, 10^{\circ} \mathrm{C}, 20$ ${ }^{\circ} \mathrm{C}, 30^{\circ} \mathrm{C}, 38^{\circ} \mathrm{C}$ and $48^{\circ} \mathrm{C}$. The microbubble solution was taken from the refrigerator at $4{ }^{\circ} \mathrm{C}$ and measured for the concentration and size distribution by optical microscopy. The microbubbles were then diluted to concentration of $3.5 \times 10^{8}$ microbubble $/ \mathrm{ml}$ 
in a $2 \mathrm{ml}$ tube with temperature equilibrated PBS buffer and then placed back into the same constant temperature water bath. $10 \mu \mathrm{L}$ of the samples was withdrawn from the tubes at periodic time intervals and distributed on a thoma cell counting chamber to measure the size and concentration of the microbubble population by optical microscopy. Images of the microbubbles were captured using a digital camera attached onto an upright microscope (Olympus $\mathrm{CH} 40$ ) with a $10 \mathrm{X}$ objective. Image analysis was performed using Image] (NIH free software). In a separate study, 9:1 DSPC/PEG ${ }_{40} \mathrm{St}$ microbubbles were produced in the same manner described above. But instead of washing with PBS at $4^{\circ} \mathrm{C}$, they were washed with PBS at the temperature to be tested and submerged in the constant temperature water bath for size and concentration measurements.

The changes in the diameter of individual microbubbles were also investigated over time using a temperature controlled hot stage at $38^{\circ} \mathrm{C}$ by observing the same frame of microscope slide without cover slip for approximately 40 minutes (Carl Zeiss Axio Imager.M2 with long working distance $63 \mathrm{X}$ objective). Time lapse images were acquired using a high resolution CCD camera (Carl Zeiss AxioCam 506 mono) and analyzed by Image] software.

\section{Langmuir Isotherms of Microbubble Shell Components}

Langmuir trough (KSV minitrough, Finland) with two movable barriers was used to investigate the phase behavior of DSPC, PEG40St monolayers and their mixture at different temperatures. The trough was thermostated at desired temperature using a circulating bath. The system was enclosed in a box to minimize possible contamination of airmonolayer-water interface and the disturbance of the monolayer by air currents. The trough was filled with ultrapure water with specific resistivity of $1.8 \times 10^{7} \Omega . \mathrm{cm}$ produced by a Millipore purification system. Cleanness of the air-water interface was confirmed by closing and opening the barriers and ensuring that the surface pressure readings do not differ by more than $\pm 0.1 \mathrm{mN} / \mathrm{m}$. The solutions of the pure components and the mixture in chloroform were prepared in screw cap septum vials at concentrations less than $1.0 \mathrm{mg} / \mathrm{ml}$ to avoid chloroform evaporation and stored at $4{ }^{\circ} \mathrm{C}$. Prior to spreading the solution, the sealed vial was taken from the refrigerator, homogenized using a bath sonicator and cooled down to room temperature under continuous stirring. The solution was withdrawn via a Hamilton microsyringe from the vial sealed tightly. The surface pressure-area $(\pi-A)$ isotherms were obtained via symmetric compression of monolayers by the two barriers. A compression speed of $5 \mathrm{~mm} / \mathrm{min}$ was used in all experiments. Each isotherm was repeated at least 4-5 times to make sure reproducibility of the isotherms. The same procedure was employed to monitor the relaxation behavior of the monolayers. The monolayers were compressed to the specified target pressure $(30 \mathrm{mN} / \mathrm{m}$ and $40 \mathrm{mN} / \mathrm{m})$ at constant temperature $\left(20^{\circ} \mathrm{C}\right.$ and $\left.38^{\circ} \mathrm{C}\right)$ and then the surface pressure was set to zero and the surface pressure evolution was followed with time over a constant area of air-water interface.

\section{RESULTS AND DISCUSSION}

\section{Preparation and Characterization of Microbubbles}

Air-filled microbubbles were prepared by sonication method from the mixture of DSPC and emulsifier $\mathrm{PEG}_{40}$ St mixed at a molar ratio of 9:1, which is the microbubble formulation commonly used in the current literature $(9,13,14,23,24)$. Sonication method was employed, which is the method used commonly for the production of microbubbles owing to its capability to provide fast production with low cost, but with some polydispersity (25). In this study, polydispersity of the microbubble solution enabled us to observe the microbubbles at different sizes at the same time. Figure 1.a shows optical microscope image of the microbubbles. Figure 1.b shows typical optical image captured under the microscope using a 10X objective and does Figure 1.c the processed micrograph created by the Image] program for concentration and size determination of the microbubbles. At least four images from different field of view were taken and analyzed for size and concentration measurements. As can be seen from the figures, the microbubbles have been achieved to be distributed quite homogeneously on the stage, allowing for determination of accurate number and size distribution of the microbubbles.

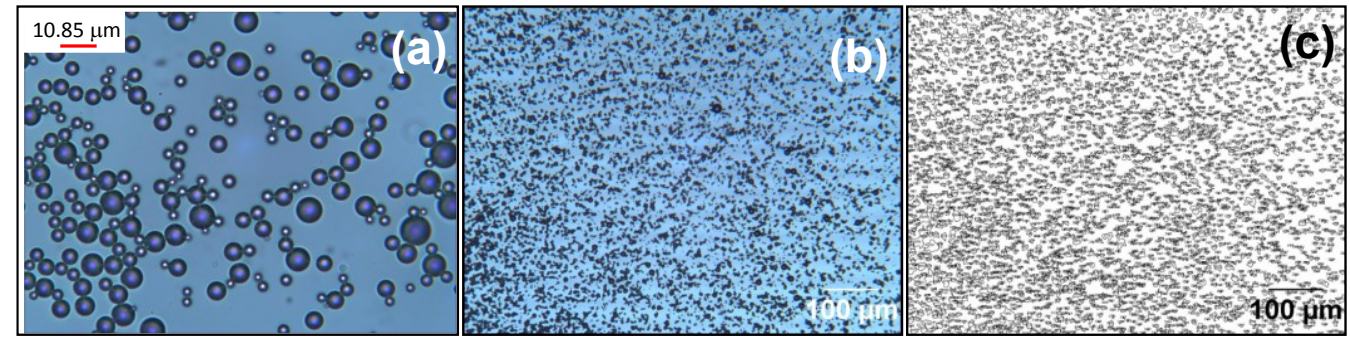

Figure 1. (a) An optical image of the microbubbles captured by $10 \mathrm{X}$ objective (b) Microscope image analysis microbubbles (c) image processed by ImageJ for size and concentration measurements. 
Effect of Temperature on Microbubble Stability The change in the concentration of the microbubbles with time at varying temperatures is shown in Figure 2.a. The numbers of the microbubbles counted at each time was determined using optical microscopy and normalized with respect to the initial number. As seen from the figure, while the microbubbles preserved their concentration at $4{ }^{\circ} \mathrm{C}$, at the other temperatures, the microbubbles maintained their concentration only up to a certain time, called 'onset time', exhibiting a plateau. The extent of the plateaus became shorter with increasing temperature. Once the onset time was reached, both the number of the bubbles started to decrease (Figure 2.a) and the mean size of the population started to increase as can be seen from the

(a)

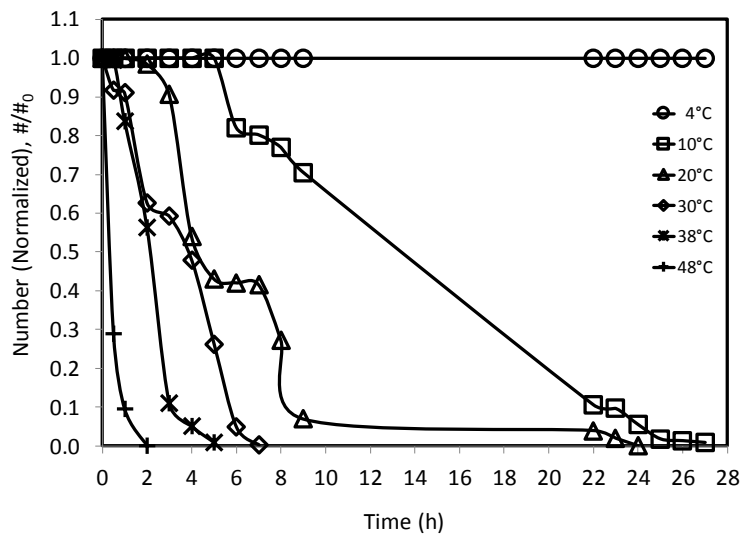

cumulative size distribution of the microbubbles at $20^{\circ} \mathrm{C}$ as an example (Figure 2.b). As seen from the figure, the initial sizes of the microbubbles were less than $9 \mu \mathrm{m}$ for the $90 \%$ of the population and exhibited a size distribution up to $14 \mu \mathrm{m}$. The size distribution of the microbubbles shifted to the right over time, indicating that the average size of the microbubbles increased in the population while the number of microbubbles decreased. The initial $d_{50 \%}$ size, which refers to the value where half of the population resides above this point, and other half resides below this point, was about $4 \mu \mathrm{m}$, and it increased to about $10 \mu \mathrm{m}$ at the end of 8 hours. The microbubbles showed similar behavior at other temperatures as well (data not shown).

(b)

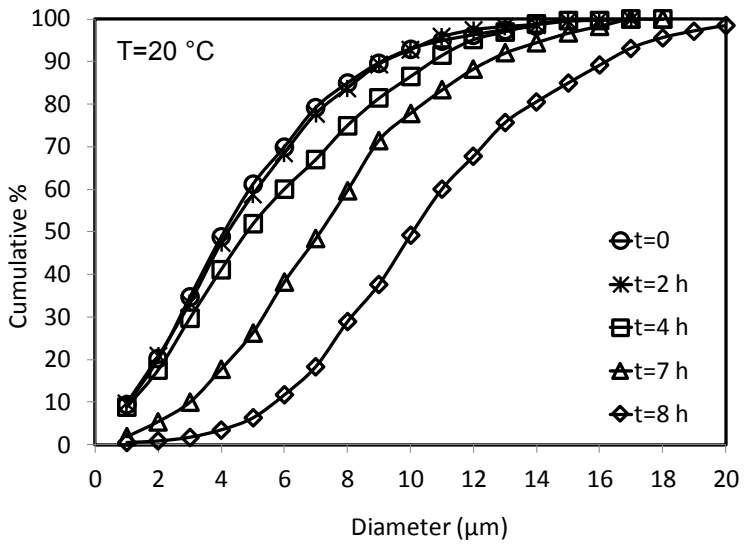

(c)

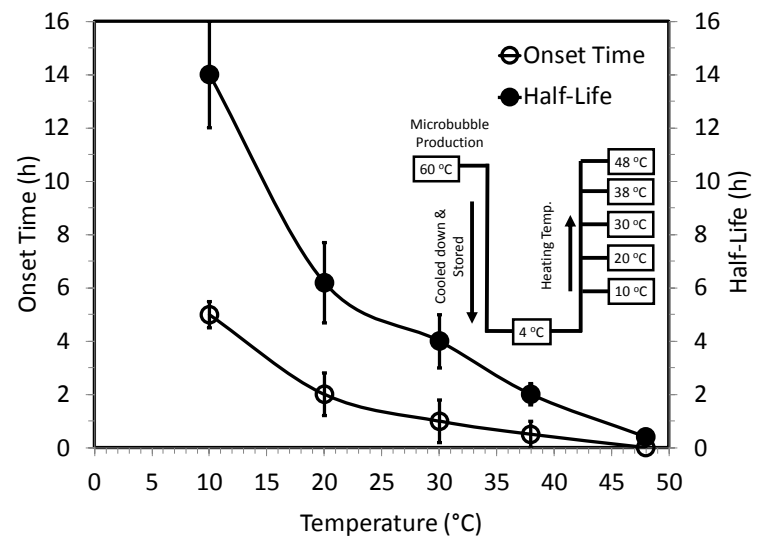

Figure 2. Change of (a) microbubble concentration (normalized with respect to initial concentration) (b) cumulative size distribution at $20^{\circ} \mathrm{C}$, (c) onset time and half-life of the 9:1 DSPC/PEG ${ }_{40}$ St microbubbles over time at different temperatures.

Figure 2.c summarizes the onset time as well as halflife of the microbubbles at different temperatures. Half-life refers to the time at which the number of microbubbles dropped to the half of their initial value. The error bars correspond to the arithmetic mean of at least three independent measurements. The microbubbles kept at $4{ }^{\circ} \mathrm{C}$ were observed to remain stable for much longer than the experimental time scale. The onset time was about 6 hours at 10 ${ }^{\circ} \mathrm{C}, 2$ hours at $20^{\circ} \mathrm{C}$ and it was shorter than an hour at elevated temperatures. Half of the population was lost at 14 hours at $10^{\circ} \mathrm{C}, 6$ hours at $20^{\circ} \mathrm{C}$ and in shorter than an hour at elevated temperatures. Both the onset time and half-life exhibited an exponential decrease with increasing temperature, similar to the observations seen with Sonovue ${ }^{\circledR}$ microbubbles (17) and $\mathrm{SF}_{6}$ microbubbles coated with $\mathrm{DSPC} / \mathrm{PEG}_{40} \mathrm{St}$ and DSPC/DSPE-PEG 2000 (9).

To understand the fate of the microbubbles in the population under the effect of temperature, the behavior of individual microbubbles was also investigated by observing the microbubble solution 
on a temperature controlled hot stage without a coverslip at $38{ }^{\circ} \mathrm{C}$. This allowed to monitor the behavior of individual microbubbles with different sizes at constant saturation and temperature. As seen from Figure 3, each microbubble had a characteristic onset time proportional to its initial size, exhibiting a continuous reduction in size beyond the onset time. The larger microbubbles maintained their initial size relatively for a longer period of time compared to the smaller ones. Evaluation of the results for population of microbubbles and individual microbubbles together suggest that the smaller microbubbles vanish earlier in the population, leaving the larger ones behind in the suspension. This explains the reason behind the increase in the mean size of the population over time after the onset time. This result is in good agreement with the Epstein-Plesset theory, suggesting that microbubble dissolution accelerates once dissolution begins and ends with a complete disappearance (26).

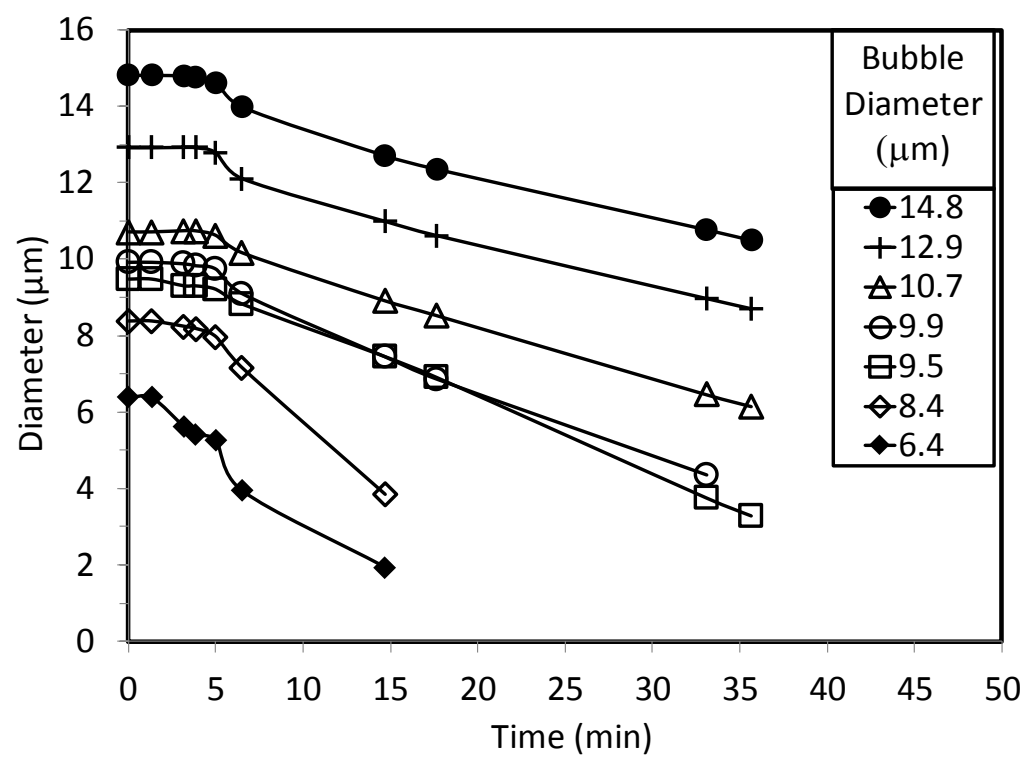

Figure 3. Changes in the diameter of individual 9:1 DSPC/PEG ${ }_{40} \mathrm{St}$ microbubbles with different sizes over time at $38^{\circ} \mathrm{C}$. Microbubbles were monitored without a cover slip at constant saturation and temperature.

\section{Langmuir Isotherms of Microbubble Shell Components}

Investigations of temperature on microbubble shell is very complex because it is not only the molecules orientations change, but also the gas dissolution can change. Langmuir monolayer technique stands out as the ideal technique to eliminate the gas dissolution effect and investigate the effect of temperature only on molecules orientation at various compression states encountered during gas dissolution. Indeed, Langmuir monolayers composed of microbubble shell components are considered as a two-dimensional "skin" of the microbubble shell (27) and therefore the technique has already been used in several studies as an experimental tool to investigate microbubble shell characteristics (2729). The technique has also been used to reveal the morphological changes in the microbubble shell with compression using the Langmuir trough in combination with fluorescence microscopy $(9,13$, 14). The Langmuir isotherms of $D S P C / P^{2} G_{40} S t$ mixture as well as the pure components were acquired at different temperatures (Figure 4). The Langmuir isotherms of DSPC is illustrated at two different temperatures, being in a good agreement with the previously published literature (30). The isotherm for DSPC at $24^{\circ} \mathrm{C}$ showed a steep rise at about $55 \mathrm{~A}^{2} /$ molecule and an extended behavior at $40^{\circ} \mathrm{C}$. However, in the low compressibility region (below $\sim 47 \mathrm{~A}^{2} /$ molecule), the isotherm at $40^{\circ} \mathrm{C}$ located to the left of the one at $24^{\circ} \mathrm{C}$. 
(a)

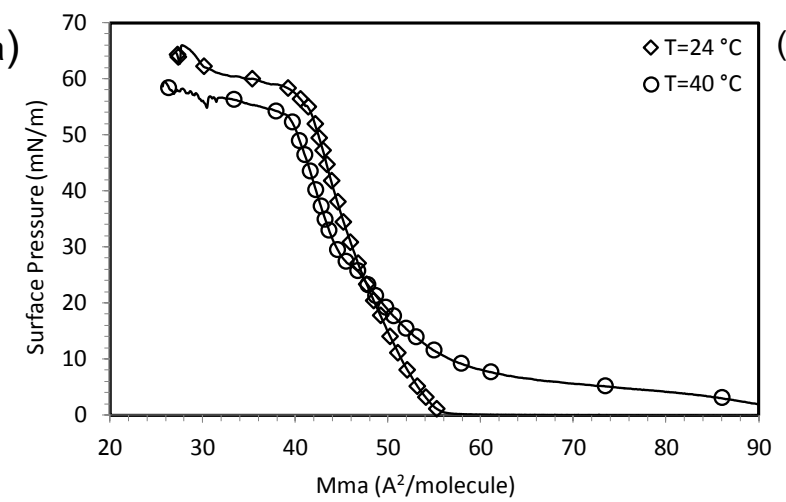

(b)

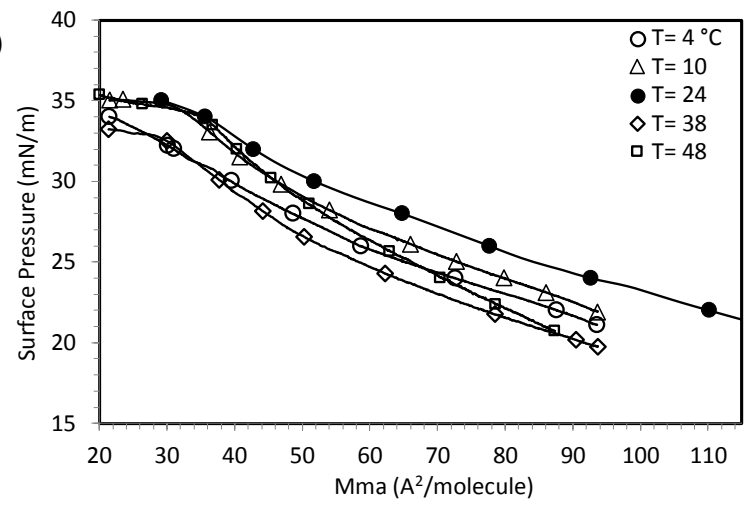

(c)

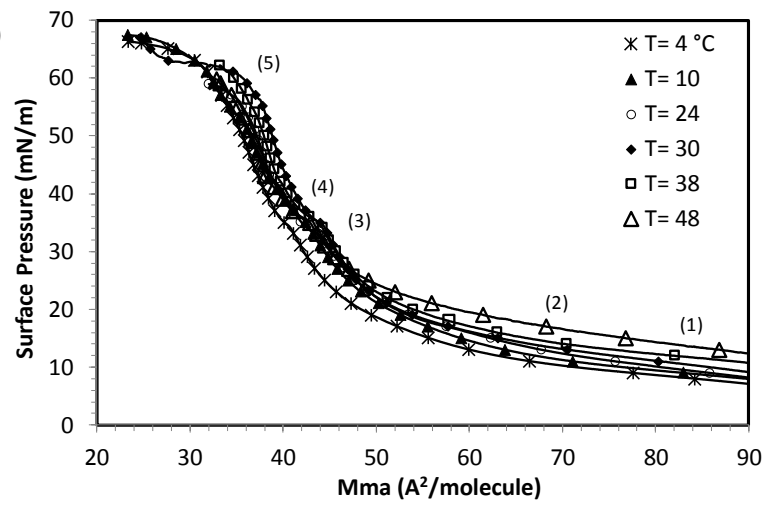

Figure 4. Langmuir isotherms of (a) DSPC, (b) PEG40St (c) $9: 1$ DSPC/PEG 40 St mixture at different temperatures. The numbers in figure (c) refers to the stage of the monolayer molecules orientation/organization depicted in Figure 5.

In comparison to DSPC monolayer, $\mathrm{PEG}_{40} \mathrm{St}$ molecules resulted in much larger surface pressures in mean molecular areas larger than $\sim 55$ $\mathrm{A}^{2} /$ molecule, exhibiting maximum surface pressures of only 33-35 $\mathrm{mN} / \mathrm{m}$ depending on the temperature, known as collapse pressure (13). The isotherms shifted to the right with increasing temperature from $4{ }^{\circ} \mathrm{C}$ to $24^{\circ} \mathrm{C}$. With further increase in temperature to $38^{\circ} \mathrm{C}$, the isotherm moved to the far left of all isotherms. Similar to $\mathrm{PEG}_{40} \mathrm{St}$ monolayer, in the high compressibility region (above $50 \mathrm{~A}^{2} /$ molecule), the mixture isotherm shifted to the right with increasing temperature, exhibiting an extended behavior. All isotherms exhibited a plateau at around 33-35 $\mathrm{mN} / \mathrm{m}$, with distinct changes at $38^{\circ} \mathrm{C}$ and $48^{\circ} \mathrm{C}$, corresponding to squeeze out of the emulsifier molecules from the monolayer (13). However, in the low compressibility region where intermolecular interactions are more dominant (below about 43 $\mathrm{A}^{2} /$ molecule), the isotherms exhibited extended behavior with increasing temperature up to $30^{\circ} \mathrm{C}$ and then shifted towards left with further increase in temperature. The numbers in Figure 4.c refers to the molecular organization state of the monolayer depicted in Figure 5.

The mean molecular areas of the isotherms at different temperatures were extracted from the Langmuir isotherms and plotted at different surface pressures to ease the visualization (Figure 6). In contrast to the insignificant changes in DSPC molecules, the changes in the mean molecular area of the pure emulsifier molecules with temperature were very prominent at all surface pressures. $\mathrm{PEG}_{40}$ St monolayer exhibited a capricious behavior with temperature, increasing first and then decreasing followed by an increase again at $48^{\circ} \mathrm{C}$ at all surface pressures. Similar behavior was observed also with the mixture. A steady increase in mean molecular area with temperature was observed for the mixture at surface pressures less than about 35 $\mathrm{mN} / \mathrm{m}$, most likely because of the $\mathrm{PEG}_{40} \mathrm{St}$ molecules not being squeezed out yet. However, above this surface pressure, the area per molecule increased with temperature up to $30^{\circ} \mathrm{C}$ and then decreased again with further increase in temperature, exhibiting more distinct changes with increasing surface pressures.

In Langmuir monolayers, higher surface pressures are observed if adsorbed molecules pack more densely at the air-water interface, i.e. possessing smaller mean molecular areas. However, in this study, achievement of the same surface pressure at higher mean molecular areas with increasing temperature can be attributed to the adsorption of increasing mass of the molecules at the interface, which can only be explained by 'curling up' of the PEG chain of the emulsifier towards the gas-liquid interface. The observed effect is more pronounced in 
pure emulsifier monolayer because the mixture contains only $10 \%$ of the emulsifier. Migration of the PEG chains of the emulsifier towards the gas-liquid interface indicates temperature dependent miscibility of PEG chains with water.

Low Temperature

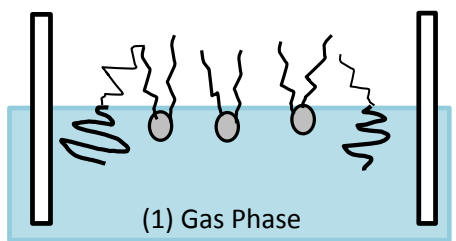

(1) Gas Phase
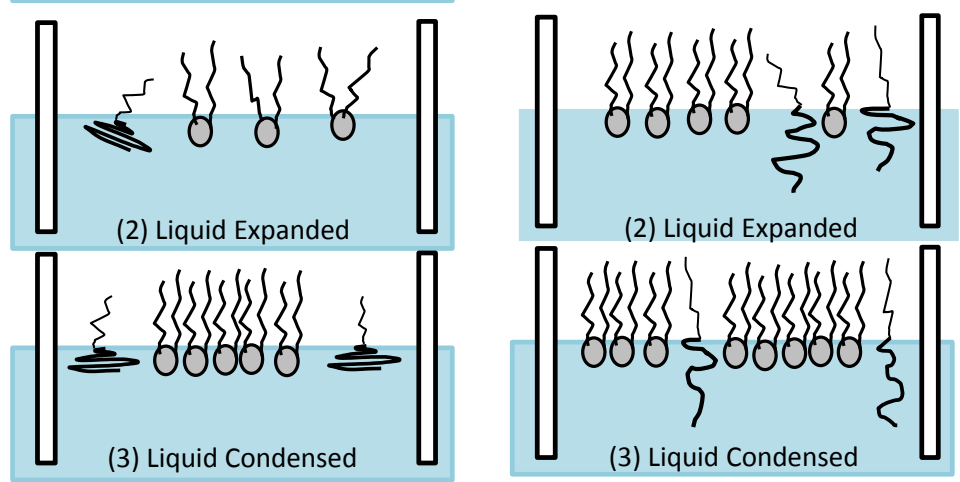

(2) Liquid Expanded

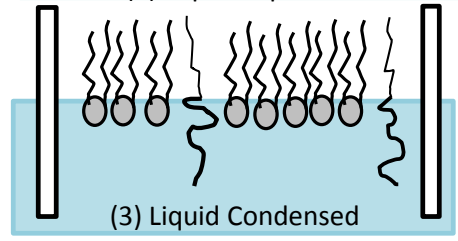

3) Liquid Condensed

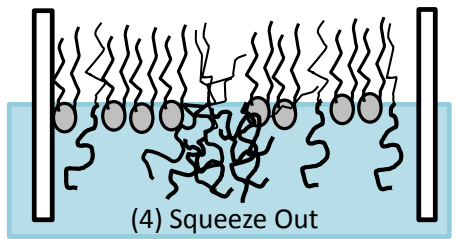

(4) Squeeze Out

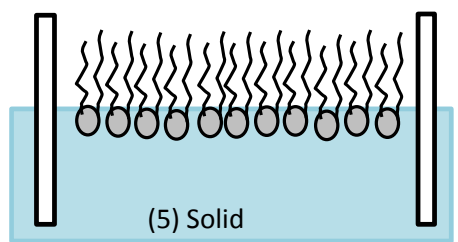

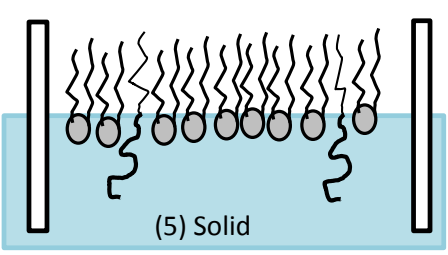

Figure 5. A cartoon depicting conformational changes and molecular organization of the microbubble shell component at various compression stages of the monolayer (as shown in Figure 4.c), mimicking the microbubble shell undergoing gas dissolution at low and high temperatures. Note that, PEG chains are more in extended into aqueous phase at lower temperatures while they curl up more towards the interface at higher temperatures.

Indeed, the interaction of ethylene oxide polymers with water occurs by means of hydrogen bonding, and the strength of the hydrogen bonds diminishes with temperature, reducing the miscibility of the PEG chains with water (31-33). Depending on the degree of miscibility, PEG chains acquire different conformations at the interface $(30,34)$, as depicted in Figure 6.b. Hence, the steady increase in the mean molecular area of the emulsifier and the mixed monolayer with increasing temperature at all surface pressures is the clear indication of decreased miscibility of PEG chains with water, indicating migration of the PEG chains towards the interface. The further decrease in the mean molecular areas indicates the local collapse of the monolayer to relieve the stress accumulated at the interface due to excessive accumulation of the PEG chains and thus increased repulsive forces between them (35). The observed effect diminished with increasing surface pressure due to reduced intermolecular spacing and associated squeeze out of the emulsifier from the interface. 
(a)

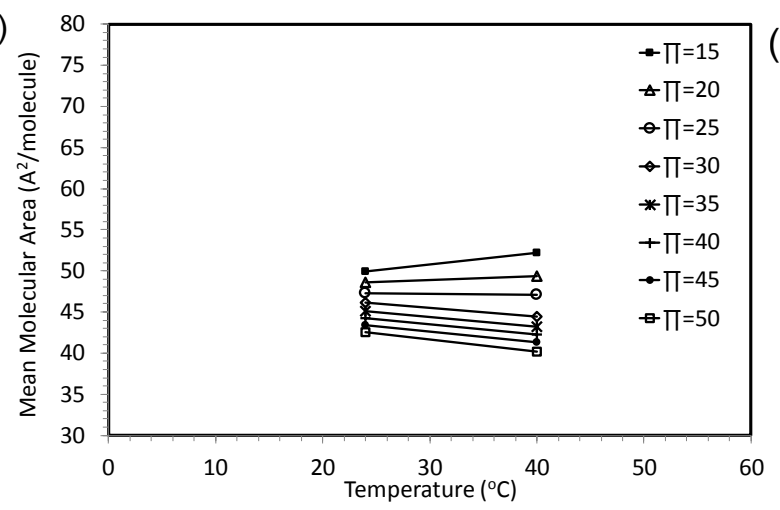

(b)

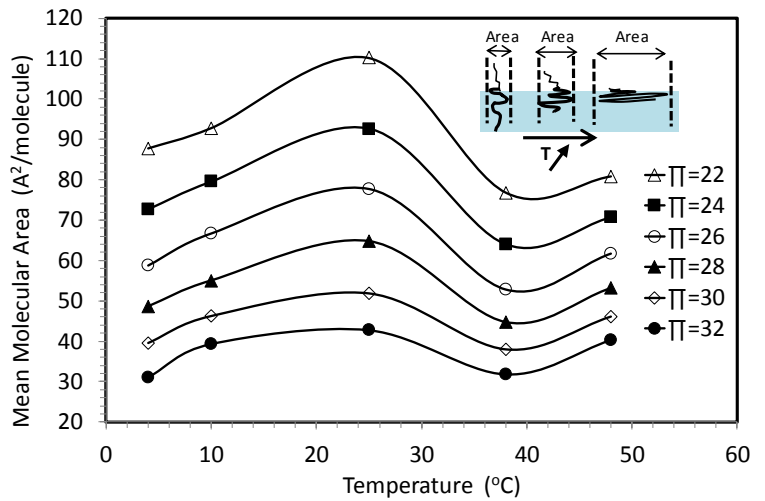

(c)

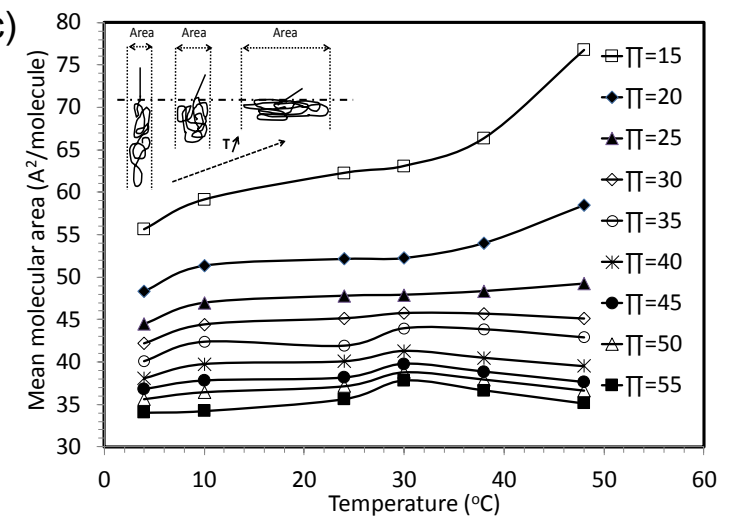

Figure 6. Change in the mean molecular area of (a) DSPC, (b) PEG40St, (c) 9:1 DSPC/PEG ${ }_{40} \mathrm{St} \mathrm{mixed}$ monolayer at different temperatures and surface pressures. The inset in figure (b) shows the conformational changes in the PEG chain of the emulsifier with temperature due to its reduced miscibility with water.

The response of the Langmuir monolayer to increased lateral interactions at constant temperature can be considered similar to the behavior of microbubble shell undergoing various degrees of compression under gas dissolution at that temperature. In order to further investigate the effects of conformational changes and increasing lateral interactions (i.e. collapse) on the microbubble shell, monolayer relaxation measurements were carried out by monitoring changes in the surface pressure of the monolayer at constant mean molecular area with time. Monolayer relaxation occurs because of the compressed monolayer normally not being in the thermodynamic equilibrium when the surface pressure is higher than the equilibrium surface pressure $(35,36)$. Monolayer relaxation behavior of the mixed monolayer was monitored at two different surface pressures (below and above the collapse pressure of the emulsifier) and at two different temperatures $\left(20^{\circ} \mathrm{C}\right.$ and $\left.38^{\circ} \mathrm{C}\right)$. The mixture isotherms showed that the surface pressure of approximately $33-35 \mathrm{mN} / \mathrm{m}$ is the collapse pressure of the emulsifier (Figure 4), in agreement with the published literature (13). As seen in Figure 7 , at $20{ }^{\circ} \mathrm{C}$, the monolayer compressed to $40 \mathrm{mN} / \mathrm{m}$ relaxes much more slowly than the monolayer at $30 \mathrm{mN} / \mathrm{m}$ (compare curves $\mathrm{b}$ and $c$ ). Because the PEG chains acquire more extended conformation into the subphase at low temperatures, during the compression of the monolayer to higher surface pressures, some portion of the emulsifier molecules become miscible with DSPC and/or trapped within DSPC domains even if the remaining portion undergoes phase separation and squeeze out, as depicted in Figure 5. Moreover, in contrast to the monolayers compressed to 30 $\mathrm{mN} / \mathrm{m}$, the initial fluctuations (see the inset in Figure 7) observed in the surface pressure of the monolayers compressed to $40 \mathrm{mN} / \mathrm{m}$ at both temperatures indicates the collapse (exclusion) of excessive emulsifier molecules, remaining temporarily associated to the surface and reinserting, but ultimately collapsing and leaving a more stable DSPC-rich monolayer behind (see Figure $5)$, as suggested elsewhere (36-38). The molecules in more ordered and rigid domains is known to collapse irreversibly (do not reinsert) (38). 


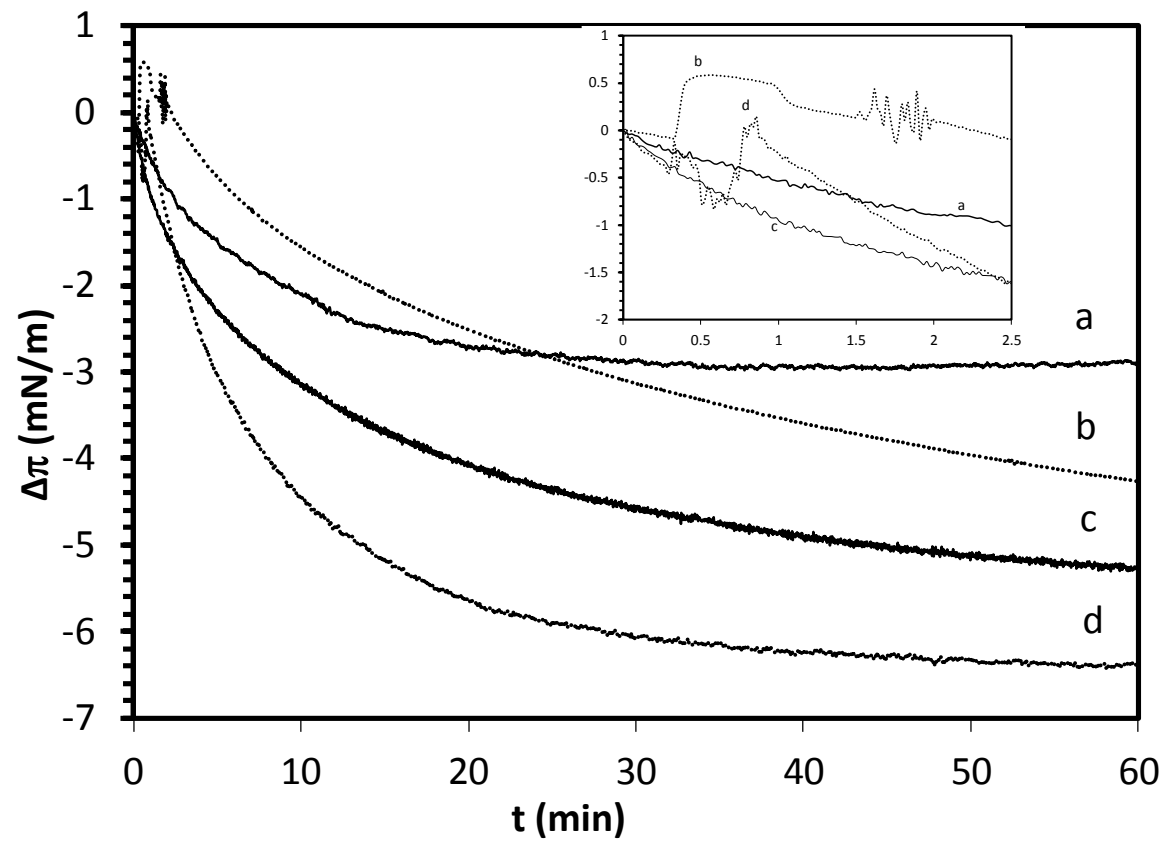

Figure 7. Change in surface pressure $(\pi)$ of 9:1 DSPC/PEG40 St mixed monolayer with time at different temperatures and target surface pressures (a) $\pi=30 \mathrm{mN} / \mathrm{m}$ and $\mathrm{T}=38{ }^{\circ} \mathrm{C}$, (b) $\pi=40 \mathrm{mN} / \mathrm{m}$ and T=20 ${ }^{\circ} \mathrm{C}$, (c) $\pi=30 \mathrm{mN} / \mathrm{m}$ and $\mathrm{T}=20^{\circ} \mathrm{C}$, (d) $\pi=40 \mathrm{mN} / \mathrm{m}$ and $\mathrm{T}=38^{\circ} \mathrm{C}$. The inset shows the initial fluctuations during 2.5 minutes.

In contrast to the monolayer at low temperature, initial decrease in the surface pressure of the monolayers compressed to $40 \mathrm{mN} / \mathrm{m}$ is much higher at $38^{\circ} \mathrm{C}$. This is because PEG chains become more hydrophobic, with higher portion of the PEG chains migrating to the interface and creating larger size fluid-like domains. As a result, the steric hindrance for accommodation of bulky PEG chains at the interface between the hydrocarbon tails of DSPC becomes much greater at higher temperatures (Figure 5). Moreover, the cohesive interactions between the hydrocarbon tails pinning the emulsifier molecules to the interface get weaker at higher temperatures to compensate for the steric repulsions between the bulky PEG chains. As a result, created fluid-like disordered domains with larger size are more prone to collapse, resulting in decrease in surface pressure at higher rates. Equilibration of the monolayer in shorter time (about $35 \mathrm{~min}$ ) in the case of higher temperature is also another indication for the exclusion of the emulsifier from the monolayer at a faster rate, leaving behind a DSPC rich monolayer.

Translation of these results to microbubbles mean that during low temperature gas core dissolution, emulsifier molecules, if not all, are trapped between DSPC molecules and some remain surface-bound upon collapse. Repetitive reinsertion of the emulsifier followed by collapse alleviates surface deformation, inhibiting gas dissolution and resulting in an onset time during microbubble dissolution. Surface deformation goes with simultaneous collapse and inplane reorganization of the molecules $(6,9)$.
Recalling that no microbubble formation is possible without the emulsifier $(10,13,39)$, the microbubble shell disintegrates when the emulsifier content decreases below a 'critical' value in the monolayer. During high temperature dissolution however, the lateral distance at the interface decreases at much higher rates under the combined effect of accumulation of more PEG chains at the interface and accelerated gas core dissolution. This results in the shell to undergo collapse with the loss of PEGylated emulsifier from the interface and disintegration at much faster rate.

In a separate study, the freshly prepared microbubbles were washed with the PBS at the target temperatures and tested immediately. The onset time curve for the microbubbles cooled down directly to the target temperature located above the curve for the microbubbles exposed to elevated temperatures from $4{ }^{\circ} \mathrm{C}$ (Figure 8). It was observed $40 \%$ increase in the onset time of microbubbles at $10{ }^{\circ} \mathrm{C}$, while $500 \%$ at $38{ }^{\circ} \mathrm{C}$. This result suggests that DSPC and emulsifier molecules are initially adsorbed around the gas bubble up to an equilibrium surface value, then upon cooling down to lower temperatures, PEG chains undergo extension into the aqueous phase due to increased hydrophilicity with decreasing temperature while the gas bubbles go to compression at the same time. Apparently, extension of the polymer chains into the aqueous phase created the space needed for the condensation of the molecules around the gas bubble while the surface of the microbubble shell was reaching a new equilibrium surface value. As a result, the emulsifier 
molecules were retained in the microbubble shell in extended conformation into the aqueous phase. This argument is also in good agreement with the observed microbubble shell morphology changes with cooling rate reported elsewhere $(13,14)$. In those studies, formation of larger dark domains surrounded by emulsifier-rich continuous phase (rich-in fluorophore and therefore brighter) during slow cooling process indicates entrapment of more emulsifier molecules between condensed phase
DSPC molecules (deficient in fluorophore and therefore darker) as results of PEG chains enabling to have enough time to extend into the aqueous phase and mix with condensed DSPC molecules, as discussed above. We also showed that conformational changes in the PEG chain of the emulsifier can be controlled by increasing the $\mathrm{PEG}_{40} \mathrm{St}$ content in the formulation, leading to production of more stable microbubbles $(40,41)$.

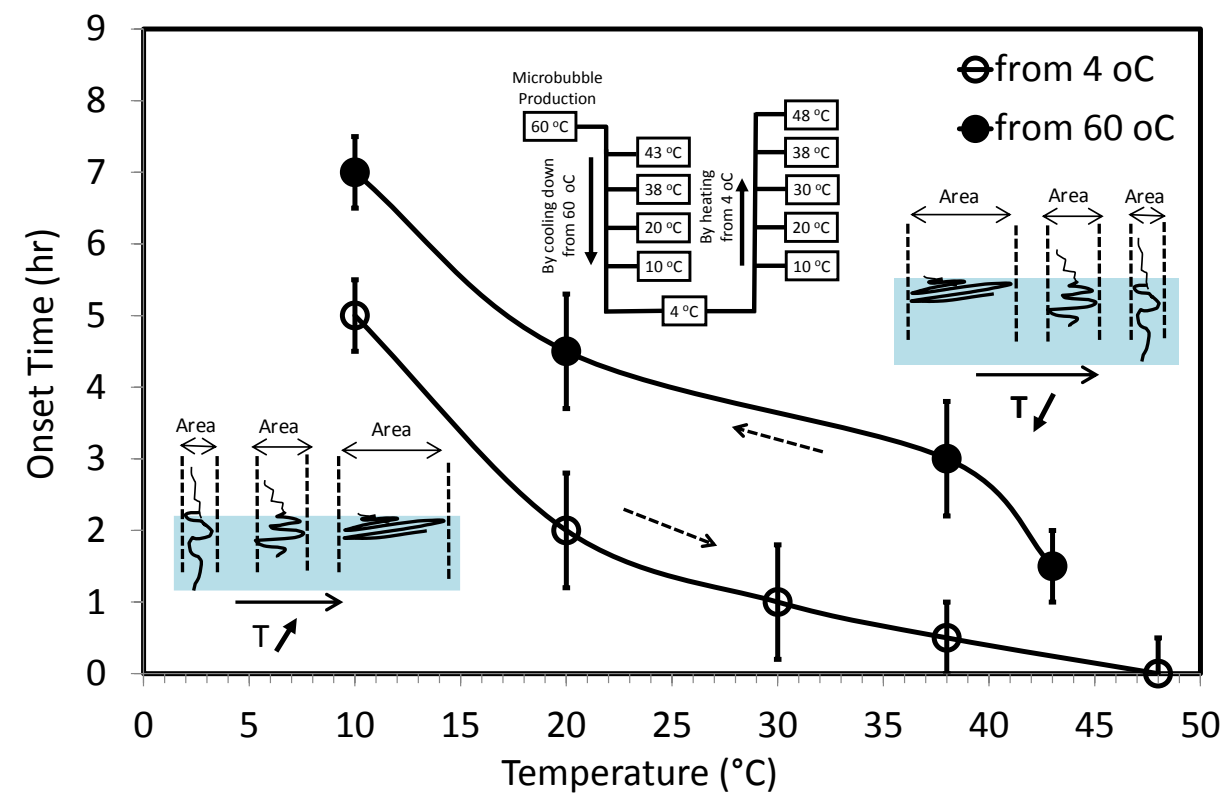

Figure 8. Comparison of the onset time of 9:1 DSPC/PEG 40 St microbubbles for two cases. In the first case (open circles), the microbubbles were cooled down to $4{ }^{\circ} \mathrm{C}$, and then exposed to elevated temperatures. In the second case (closed circles), the microbubbles were directly cooled down from elevated temperature to target temperature and tested immediately.

\section{CONCLUSIONS}

This study demonstrated that the conformational changes induced by temperature in the PEG chain of the emulsifier have important influence on destabilization of microbubbles. Upon their production, microbubbles are usually refrigerated and then exposed to higher temperatures for administration and/or laboratory investigations. Langmuir isotherms and surface pressure relaxation measurements demonstrated that PEG chain of the emulsifier became predominantly hydrophobic with increasing temperature, curling up towards the airwater interface. Accordingly, surface deformations initiates on the microbubble shell. With the initiation of surface deformations and subsequently gas dissolution, microbubble shell undergoes compression, causing lateral distance between the shell components to decrease. At the point where the monolayer exceeds the equilibrium surface pressure value, the shell/monolayer undergoes collapse mostly in the emulsifier rich regions, as evidenced by the reinsertions (i.e. fluctuations) in the monolayer relaxation experiments. Repetitive reinsertion of the emulsifier gives rise to the onset time, which is a period of time during which the microbubbles preserve their stability and then exhibit a decrease in total number by earlier disappearance of the smaller microbubbles from the population. When combined with the gas dissolution at a faster rate at higher temperatures, the more hydrophobic the PEG chains, the more the surface deformations, resulting in more destructive collapse of the monolayer and lower stability of microbubbles. It was shown that microbubble stability can be improved by controlling the conformational changes in the PEG chain of the emulsifier by cooling down the microbubbles to desired temperature upon their production. As we have shown before, the another way to control conformational changes in the PEG chain of the emulsifier is to increase the $\mathrm{PEG}_{40}$ St content in the formulation $(40,41)$. Overall, the results suggest that the effect of the conformational changes induced by temperature in the PEG chain of the emulsifier should be taken into account for the design of more stable microbubbles. 


\section{ACKNOWLEDGEMENTS}

The author would like to thank The Scientific and Technological Research Council of Turkey (TUBITAK) for the financial support through the project number of 109M494. The author also would like to thank Elif Seniz Bolukcu and Emine Aysu Sagdic for their help in part of the experiments.

\section{REFERENCES}

1. Paefgen $V$, Doleschel $D$, Kiessling $F$. Evolution of contrast agents for ultrasound imaging and ultrasound-mediated drug delivery. Front Pharmacol. 2015;6.

2. Lentacker I, Geers B, Demeester J, De Smedt SC, Sanders NN. Design and Evaluation of Doxorubicin-containing Microbubbles for Ultrasoundtriggered Doxorubicin Delivery: Cytotoxicity and Mechanisms Involved. Molecular Therapy. 2010;18(1):101-8.

3. Borden MA, Longo ML. The dependence of lipid-coated microbubble dissolution behavior on acyl chain length. Biophys J. 2002;82(1):35a-a.

4. Garg S, Thomas AA, Borden MA. The effect of lipid monolayer in-plane rigidity on in vivo microbubble circulation persistence. Biomaterials. 2013;34(28):6862-70.

5. Kwan JJ, Borden MA. Lipid monolayer dilatational mechanics during microbubble gas exchange. Soft Matter. 2012;8(17):4756-66.

6. Pu G, Borden MA, Longo ML. Collapse and shedding transitions in binary lipid monolayers coating microbubbles. Langmuir. 2006;22(7):29939.

7. Kabalnov A, Klein D, Pelura T, Schutt E, Weers J. Dissolution of multicomponent microbubbles in the bloodstream: 1. Theory. Ultrasound Med Biol. 1998;24(5):739-49.

8. Borden MA, Longo ML. Oxygen permeability of fully condensed lipid monolayers. J Phys Chem B. 2004;108(19):6009-16.

9. Kwan JJ, Borden MA. Lipid monolayer collapse and microbubble stability. Advances in Colloid and Interface Science. 2012;183:82-99.

10. Shen $Y$, Powell RL, Longo ML. Interfacial and stability study of microbubbles coated with a mono stearin/monopalmitin-rich food emulsifier and PEG40 stearate. Journal of Colloid and Interface Science. 2008;321(1):186-94.

11. Shen $Y Y$, Powell RL, Longo ML. Influence of the dissolution rate on the collapse and shedding behavior of monostearin/monopalmitin-rich coated microbubbles. Langmuir. 2008;24(18):10035-40.

12. Kwan JJ, Borden MA. Microbubble Dissolution in a Multigas Environment. Langmuir. 2010;26(9):6542-8.

13. Borden MA, Pu G, Runner GJ, Longo ML. Surface phase behavior and microstructure of lipid/PEG-emulsifier monolayer-coated microbubbles. Colloid Surface B. 2004;35(3-4):20923.

14. $\mathrm{Pu} \mathrm{G}$, Longo $\mathrm{ML}$, Borden MA. Effect of microstructure on molecular oxygen permeation through condensed phospholipid monolayers. Journal of the American Chemical Society. 2005;127(18):6524-5.

15. Petrache HI, Dodd SW, Brown MF. Area per lipid and acyl length distributions in fluid phosphatidylcholines determined by $\mathrm{H}-2$ NMR spectroscopy. Biophys J. 2000;79(6):3172-92.

16. Kucerka N, Nieh MP, Katsaras J. Fluid phase lipid areas and bilayer thicknesses of commonly used phosphatidylcholines as a function of temperature. Biochimica Et Biophysica Acta-Biomembranes. 2011;1808(11):2761-71.

17. Mulvana H, Stride E, Hajnal JV, Eckersley RJ. Temperature Dependent Behavior of Ultrasound Contrast Agents. Ultrasound Med Biol. 2010;36(6):925-34.

18. Mulvana $H$, Stride E, Tang MX, Hajnal JV, Eckersley R. Temperature-Dependent Differences in the Nonlinear Acoustic Behavior of Ultrasound Contrast Agents Revealed by High-Speed Imaging and Bulk Acoustics. Ultrasound Med Biol. 2011;37(9):1509-17.

19. Swanson EJ, Mohan V, Kheir J, Borden MA. Phospholipid-Stabilized Microbubble Foam for Injectable Oxygen Delivery. Langmuir. $2010 ; 26(20): 15726-9$.

20. Farook $U$, Stride $E$, Edirisinghe $M J$. Preparation of suspensions of phospholipid-coated microbubbles by coaxial electrohydrodynamic atomization. Journal of the Royal Society Interface. 2009;6(32):271-7.

21. Borden MA, Longo ML. Dissolution behavior of lipid monolayer-coated, air-filled microbubbles: Effect of lipid hydrophobic chain length. Langmuir. 2002;18(24):9225-33.

22. Grant CA, McKendry JE, Evans SD. Temperature dependent stiffness and visco-elastic behaviour of lipid coated microbubbles using atomic force microscopy. Soft Matter. 2012;8(5):1321-6. 
23. Borden M. Nanostructural features on stable microbubbles. Soft Matter. 2009;5(4):716-20.

24. Upadhyay A, Dalvi SV, Gupta G, Khanna N. Effect of PEGylation on performance of protein microbubbles and its comparison with lipid microbubbles. Mat Sci Eng C-Mater. 2017;71:42530.

25. Stride $\mathrm{E}$, Edirisinghe M. Novel preparation techniques for controlling microbubble uniformity: a comparison. Medical \& Biological Engineering \& Computing. 2009;47(8):883-92.

26. Epstein PS, Plesset MS. On the Stability of Gas Bubbles in Liquid-Gas Solutions. J Chem Phys $1950 ; 18(11): 1505-9$.

27. Wang WH, Moser CC, Wheatley MA. Langmuir trough study of surfactant mixtures used in the production of a new ultrasound contrast agent consisting of stabilized microbubbles. J Phys ChemUs. $1996 ; 100(32): 13815-21$.

28. Abou-Saleh RH, Peyman SA, Johnson BRG, Marston G, Ingram N, Bushby R, et al. The influence of intercalating perfluorohexane into lipid shells on nano and microbubble stability. Soft Matter. 2016;12(34):7223-30.

29. Xing ZW, Ke HT, Wang JR, Zhao B, Yue XL, Dai $Z F$, et al. Novel ultrasound contrast agent based on microbubbles generated from surfactant mixtures of Span 60 and polyoxyethylene 40 stearate. Acta Biomaterialia. 2010;6(9):3542-9.

30. Chou TH, Chu IM. Thermodynamic characteristics of DSPC/DSPE-PEG(2000) mixed monolayers on the water subphase at different temperatures. Colloid Surface B. 2003;27(4):33344.

31. Bae YC, Lambert SM, Soane DS, Prausnitz JM. Cloud-Point Curves of Polymer-Solutions from Thermooptic Measurements. Macromolecules. $1991 ; 24(15): 4403-7$.

32. Gao X, Kucerka N, Nieh MP, Katsaras J, Zhu $\mathrm{SP}$, Brash JL, et al. Chain Conformation of a New
Class of PEG-Based Thermoresponsive Polymer Brushes Grafted on Silicon as Determined by Neutron Reflectometry. Langmuir. 2009;25(17):10271-8.

33. Tyrode $E$, Johnson CM, Rutland MW, Claesson PM. Structure and hydration of poly(ethylene oxide) surfactants at the air/liquid interface. A vibrational sum frequency spectroscopy study. Journal of Physical Chemistry C. 2007;111(31):11642-52.

34. Frey SL, Lee KYC. Temperature dependence of poloxamer insertion into and squeeze-out from lipid monolayers. Langmuir. 2007;23(5):2631-7.

35. Jebrail $M$, Schmidt $R$, DeWolf $C E$, Tsoukanova $V$. Effect of aliphatic chain length on stability of poly(ethylene glycol)-grafted phospholipid monolayers at the air/water interface. Colloid Surface A. 2008;321(1-3):168-74.

36. Angelova A, De Coninck J, Ionov R. Equilibrium surface properties of lipid mixtures of retinal, phosphatidylcholine and fatty acid derivatives at the air/water interface. Supramol Sci. $1997 ; 4(3-4): 207-14$.

37. Gopal A, Lee KYC. Morphology and collapse transitions in binary phospholipid monolayers. J Phys Chem B. $2001 ; 105(42): 10348-54$.

38. Lipp MM, Lee KYC, Takamoto DY, Zasadzinski JA, Waring AJ. Coexistence of buckled and flat monolayers. Physical Review Letters. $1998 ; 81(8): 1650-3$.

39. Hristova K, Kenworthy A, Mcintosh TJ. Effect of Bilayer Composition on the Phase-Behavior of Liposomal Suspensions Containing Poly(Ethylene Glycol)-Lipids. Macromolecules. 1995;28(23):76939.

40. Kilic S, Bolukcu ES. Phase behavior of DSPC/PEG(40)St mixtures at higher emulsifier contents. Colloid Surface B. 2018;171:368-76.

41. Kilic S. Quantification of PEG(40)St squeeze out from DSPC/PEG(40)St monolayers at higher molar ratios. Colloid Surface A. 2018;551:58-64. 